\title{
Restricción del riego en la producción de biomasa del pasto Pennisetum sp
}

\section{Restriction of irrigation in the production of pasture biomass Pennisetum sp}

\author{
${ }^{\bullet}$ Jesús Enrique Chavarría Párraga ${ }^{1}$, Wilmer Omar Pilaloa David ${ }^{2}$, Carlos Enrique González Arteaga ${ }^{1}$ \\ Luis Enrique Párraga Muñoz ${ }^{3}$ \\ ${ }^{1}$ Pontificia Universidad Católica del Ecuador Sede Manabí. Carrera Ingeniería Agroindustrial. Campus Chone, vía a Quito \\ Km.11.5, Chone.jchavarria@pucem.edu.ec; cgonzalez@pucem.edu.ec \\ ${ }^{2}$ Universidad Agraria del Ecuador. Av. 25 de Julio y Pio Jaramillo. P.O. BOX 09-04-100. wilmerppp@hotmail.com \\ ${ }^{3}$ Escuela Superior Politécnica Agropecuaria de Manabi "Manuel Félix López" 10 de Agosto 82 y Granda Centeno. Calceta. \\ Cantón Bolivar, Manabi. lenrique1961@hotmail.com
}

Rec.: 31.05.2017. Acept.: 01.09.2017. Publicado el 1 de diciembre de 2017

Resumen

T a investigación se desarrolló en el sitio Garrapata, ¿Parroquia Santa Rita, provincia Manabí; durante la época seca del año 2015. El estudio consistió en evaluar la restricción hídrica en la producción del pasto king grass morado (Pennisetum sp) bajo riego por aspersión. Se utilizó un diseño de bloques completos al azar, formado de tres tratamientos y seis repeticiones. Los tratamientos consistieron en aplicar tres láminas de reposición de agua 80\% ETc, 100\% ETc, $120 \%$ ETc, calculados a partir de los registros diarios de evaporación en un tanque evaporímetro tipo A y afectados por el coeficientes Kp (Coeficiente de tina, método FAO $33,1980)$. Las variables evaluadas fueron altura de planta a los 15 y 30 días del corte de igualación del pasto, altura de planta a la cosecha $(\mathrm{m})$, rendimiento de forraje verde por parcela y hectárea $(\mathrm{kg})$ y materia seca $(\%)$. Los resultados indican que el tratamiento lámina de riego al 120\% de la ETc (T3), respondió de manera favorable en altura de planta a la cosecha $(2.08 \mathrm{~m})$, rendimiento (53000 kg.ha-1 $)$; el tratamiento lámina de riego al $100 \%$ de la ETc (T2) obtuvo mayor contenido de materia seca $(19.86 \%)$. El pasto king grass morado regado con una lámina de riego al 120\% de la ETc, constituye una modalidad que mejora el rendimiento productivo y rentable del cultivo y podría aplicarse en la zona.

Palabras clave: pasto de corte, rendimiento de forraje verde, lámina de riego en pasto.
$T$ he research was conducted at the site Garrapata, Parroquia Santa Rita, Manabi province; during the dry season of 2015The study consisted in evaluating the water restriction in the production of purple king grass (Pennisetum $\mathrm{sp}$ ) under aspersion irrigation method. A design randomized complete, consisting of three treatments and six repetitions blocks were used. The treatments were applied three sheets of water replenishment $80 \%$ ETc 100\% ETc, 120\% ETc calculated from daily records of evaporation obtained from pan evaporation type A and affected by the coefficients Kp (coefficient tub, method FAO 33,1980). The variables evaluated were: plant height at 15 and 30 days equalization cut grass, plant height at harvest $(\mathrm{m})$, green forage yield per plot and hectare $(\mathrm{kg})$ and dry matter $(\%)$. The results show the irrigation treatment sheet of $120 \%$ ETc (T3), responded favorably in plant height at harvest $(2.08 \mathrm{~m})$, Yield $\left(53000 \mathrm{kgha}^{-1}\right)$; the blade irrigation treatment $100 \%$ ETc (T2) obtained higher dry matter content $(19.86 \%)$. Purple grass king grass watered with irrigation depth to $120 \%$ of ETc, is a modality that improves productive and profitable crop yield and could be applied in the area.

Key words: grass cutting, green forage yield, grass blade irrigation. 
Introducción

E las últimas décadas, la agricultura y, específicamente, los sistemas de producción ganadera, se han convertido en uno de los procesos productivos más afectados por la influencia del medio ambiente (Rodríguez et al., 2008).

En la actualidad, el uso intensivo de pastos para corte debe considerarse como una herramienta de bajo costo, para incrementar la producción del ganado. Esto implica minimizar el desperdicio de forraje eliminando el pisoteo, evitando el gasto de energía durante el pastoreo (Dávila y Urbano, 2005).La irrigación es responsable por el 80\% del agua utilizada en Ecuador. La demanda por agua de irrigación es alta y aumenta anualmente, especialmente en la región de los Andes y en las planicies costeras áridas (ECUADORCEPAL, 2012).

El uso de la irrigación en ambientes semiáridos es una técnica indispensable para reducir la deficiencia en la producción de forraje en el período de déficit hídrico. Diversos autores verificaron efectos positivos de la irrigación sobre la productividad de forrajes tropicales (Dourato-Neto et al., 2002; Gargantini et al., 2005; Souza et al., 2005).

Últimamente, ha habido un uso creciente del riego a fin de aumentar la capacidad productiva de la biomasa de forrajes tropicales. Sin embargo, la respuesta productiva de los pastizales, depende del riego, pero también está directamente relacionada con factores climáticos, especialmente la temperatura y el fotoperíodo (Alencar et al., 2009).

El paso continuo del agua por las plantas, permite que éstas mantengan los estomas abiertos sin que se deshidraten sus tejidos, y a la vez, una pequeña parte de esta agua es fijado mediante la fotosíntesis en la elaboración de materia orgánica, junto al $\mathrm{CO}_{2}$ que las plantas toman por los estomas. Si la cantidad de agua que hay en el suelo es limitante para la planta, ésta también realiza su propia regulación mediante el cierre estomático (Idso, 1983).

La asignación de asimilados es el resultado del crecimiento y desarrollo, los cuales son mutuamente dependientes y son difíciles de analizar en experimentos separados. Los tallos laterales, por ejemplo, no se desarrollan cuando hay una escasez de carbohidratos disponibles, en ese caso, el desarrollo es restringido por el crecimiento. Además, la asignación de asimilados resulta de la interacción de condiciones climáticas, prácticas culturales y genotipo (Ñústez et al., 2009).

Una de los factores limitantes en la producción de biomasa de los pastos tropicales es el riego, por tal motivo, el estudio tiene como objetivo evaluar la aplicación de diferentes láminas de reposición de agua, consideradas en base a la ETc y su relación en la producción del pasto king grass morado (Pennisetum sp).

\section{Materiales y métodos}

T a investigación se realizó en el sitio Garrapata del cantón Chone, provincia de Manabí, geográficamente localizada en las siguientes coordenadas $0^{\circ} 39^{\prime} 34^{\prime \prime}$ Latitud Sur; y $80^{\circ}$ 02 ' 45.07" Longitud Oeste y una altitud de $22 \mathrm{msnm}$, la temperatura media anual es $25.62{ }^{\circ} \mathrm{C}$, la humedad relativa es $90 \%$, la precipitación $987.85 \mathrm{~mm}$ y la evaporación 1112.88 $\mathrm{mm}$.

El trabajo experimental se efectuó entre los meses julio-diciembre, el factor en estudio fue láminas de riego correspondientes a 120,100 y $80 \%$ de la evapotranspiración del cultivo ETc durante todo el ensayo, calculado mediante el tanque o bandeja evaporimétrica clase A. Se utilizó el diseño de bloques completos al azar, con tres tratamientos y seis repeticiones, y un total de 18 unidades experimentales.

El área de las parcelas fue de diez metros de ancho por diez metro de largo con un área de $1950 \mathrm{~m}^{2}$ para todo el experimento. El cálculo de la Etc se realizó con las siguientes formulas:

Eto $=E v * \mathrm{Kp}$

Eto es la evaporación del cultivo de referencia, expresada en mm por día, Ev es la evaporación de la cubeta, expresada en mm por día y Kp es el coeficiente de la cubeta de la clase A (Malek, 1987; Lage et al., 2003).

\section{Etc $=$ Eto $* \mathrm{Kc}$}

Etc es la evapotranspiración del cultivo o real, expresada en $\mathrm{mm}$ por día y $\mathrm{Kc}$ es el coeficiente del cultivo (Navejas et al., 2011). Obtenido el valor de Etc se ajustaba según los tratamientos en estudio (Cuadro 1).

La parcela de pasto se estableció en el mes de enero del mismo año, aprovechando las precipitaciones de la época lluviosa, se sembró a un distanciamiento de $1 \mathrm{~m}$ entre planta y entre hileras. En el mes de mayo se instaló el sistema de riego por aspersión a las parcelas de pastos y en julio se realizó el corte de igualación del pasto para iniciar el ensayo.

Para la variable altura de planta se utilizó 10 plantas tomados al azar de la parcela útil de cada tratamiento, se midió altura desde la parte basal hasta el extremo final de la hoja del ápice (m) a los 15, 30 días y a la cosecha. La variable rendimiento forraje verde se evaluó al momento de la cosecha, es decir, a los 45 días, utilizando la metodología del metro cuadrado, la cual consistió en seleccionar en la unidad experimental una porción de $1 \mathrm{~m}^{2}$ con la ayuda de un flexómetro, se cosechó todo el pasto, se utilizó una balanza para determinar su peso en $\mathrm{kg}$. Se tomaron 4 porciones de 1 $\mathrm{m}^{2}$ por unidad experimental. Para obtener el porcentaje de materia seca, se utilizó una muestra del pasto cosechado por 
Cuadro 1. Tiempo en las etapas y coeficiente del cultivo de pasto king grass morado después del corte de igualación del pasto

\begin{tabular}{lcc}
\hline Etapas & Kc & Tiempo (días) \\
\hline I & 0.39 & 15 \\
II & 0.42 & 15 \\
III & 1.02 & 15 \\
\hline
\end{tabular}

Herrera et al. (2010)

cada tratamiento, se picó y almacenó en una bolsa de papel para que no pierda humedad, luego se pesó y se introdujo en la estufa a una temperatura de $100^{\circ} \mathrm{C}$ por 24 horas para hallar la materia seca, finalmente se tomó el peso del pasto seco.

Se realizó análisis de varianza y las diferencias entre medias se realizaron mediante la prueba de Tukey $(\mathrm{p}<0.05)$, además se determinó la correlación y regresión entre las variables evaluadas.

\section{Resultados y discusión}

$\mathrm{L}$ a altura de planta a los 30 días, presentó la existencia de variación significativa entre los tratamientos $(\mathrm{p}<0.05)$, donde la lámina de riego al 120\% de la ETc, prevalece con el mejor promedio con $1.26 \mathrm{~m}$ por planta, siendo estadísticamente igual a la lámina de riego al 100\% de la ETc, mientras, el promedio menor lo consiguió la lámina de riego al $80 \%$ de la ETc, con una altura por planta de $1.09 \mathrm{~m}$ (Cuadro 2). La altura de planta a la cosecha, determinó la existencia de variación altamente significativa entre los tratamientos $(\mathrm{p}<0.01)$, donde la lámina de riego al $120 \%$ de la ETc, prevalece con el mejor promedio con $2.08 \mathrm{~m}$ por planta, el promedio menor lo consiguió la lámina de riego al $80 \%$ de la ETc, con una altura por planta de $1.65 \mathrm{~m}$.

El crecimiento vegetativo del pasto king grass morado fue influenciado por el incremento de las láminas de riego y se mostró a los 30 días, debido a que en los primeros quince días el pasto tenía poca cantidad de hojas, constituyendo la principal limitación para el crecimiento de un cultivo al comienzo de su brotación. Por ello, la mayor altura a los 30 días del corte de igualación y a la cosecha del pasto king grass morado fue con la lámina de riego al 120\% de la ETc, por tanto, el pasto tenia agua suficiente para cumplir sus funciones fisiológicas normales y por ende crecer más, resultados que coinciden por lo manifestado por Ribas et al. (1987) quienes manifiestan que las pasturas al tener agua almacenada en el suelo en cantidades suficientes pueden incrementar su crecimiento vegetativo. Además, coincide con Dourato-Neto et al. (2002), Gargantini et al. (2005) y Souza et al. (2005) quienes manifiestan que, la irrigación tiene efectos significativos sobre la productividad
Cuadro 2. Promedio de altura de planta (m) a los 15,30 días y a la cosecha (45 días) del pasto king grass morado

\begin{tabular}{llll}
\hline \multirow{2}{*}{ Tratamientos } & \multicolumn{3}{c}{ Altura de planta (m) } \\
\cline { 2 - 4 } & 15 días & 30 días & Cosecha \\
\hline $\begin{array}{l}\text { Lámina de riego al 80\% } \\
\text { de la ETc }\end{array}$ & $0.537 \mathrm{a}$ & $1.09 \mathrm{~b}$ & $1.65 \mathrm{~b}$ \\
$\begin{array}{l}\text { Lámina de riego al 100\% } \\
\text { de la ETc }\end{array}$ & $0.542 \mathrm{a}$ & $1.14 \mathrm{ab}$ & $1.79 \mathrm{~b}$ \\
$\begin{array}{l}\text { Lámina de riego al 120\% } \\
\text { de la ETc }\end{array}$ & $0.545 \mathrm{a}$ & $1.26 \mathrm{a}$ & $2.08 \mathrm{a}$ \\
\hline CV $(\%)$ & 1.64 & 8.5 & 9.04 \\
\hline
\end{tabular}

Medias seguidas con la misma letra, no difieren estadísticamente (Tukey, $\mathrm{p}>0.05$ )

de los forrajes tropicales y en las condiciones del semiárido es una técnica indispensable para reducir la deficiencia en la producción de forraje en el período de déficit hídrico.

El rendimiento de forraje verde por parcela y por hectárea, determinó la existencia de variación significativa entre los tratamientos $(\mathrm{p}<0.05)$ donde la lámina de riego al $120 \%$ de la ETc, prevalece con el mejor promedio de $530 \mathrm{~kg}$ por parcela y $53000 \mathrm{~kg}$ por hectárea respectivamente, siendo estadísticamente igual a la lámina de riego al $100 \%$ de la ETc, mientras, el promedio menor lo consiguió la lámina de riego al $80 \%$ de la ETc, con un rendimiento por parcela de 458.33 y $45833.33 \mathrm{~kg}$ por hectárea (Figura 1).

El rendimiento en forraje verde del pasto king grass morado estuvo influenciado por el incremento de las láminas de riego, al conseguirse una adecuada aportación de agua al pasto y para cumplir sus funciones fisiológicas normales. La respuesta del cultivo fue incrementar la producción, resultados que coinciden con lo dicho por Doorembos y Kazan (1986) quienes manifiestan que los rendimientos de las pasturas con riego, incrementa su producción considerando el volumen de agua que se les aplique, lo cual minimiza los riegos de las inversiones agropecuarias. Además, coincide con Alencar et al. (2009), el aumento de la capacidad

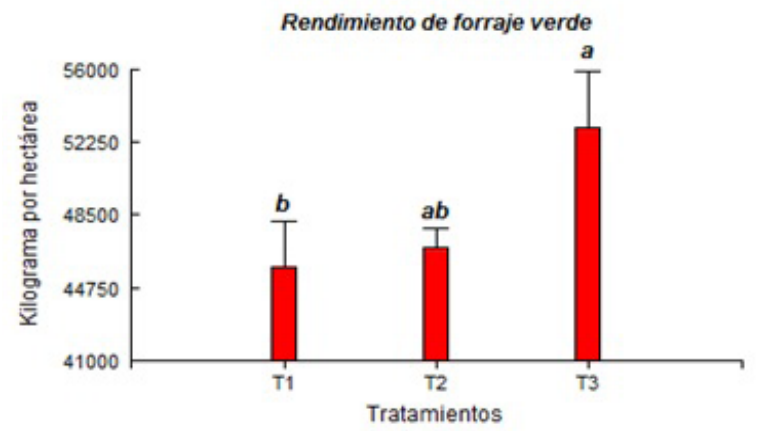

Figura 1. Rendimiento de forraje verde del pasto king grass morado para los diferentes tratamientos (T1: Lámina de riego al $80 \%$ de la ETc. T2: Lámina de riego al $100 \%$ de la ETc. T3: Lámina de riego al $120 \%$ de la ETc.). Barras seguidas con la misma letra, no difieren estadísticamente (Tukey, $\mathrm{p}>0.05$ ) 
productiva de la biomasa de forrajes tropicales depende del riego y está directamente relacionada con factores climáticos, especialmente la temperatura y el fotoperíodo.

La materia seca (MS) del pasto king grass morado no presentó variación significativa entre los tratamientos (Cuadro 3 ), esto quiere decir, las láminas de riego no influyeron en el contenido de MS del pasto, debido a la condición de humedad en el suelo presente en el período de evaluación, que favoreció la expresión del potencial de producción de materia seca (MS) del pasto. Esta disponibilidad de humedad genera en las plantas que expresen su potencial productivo, en este caso, en la producción de MS, resultados que contrastan con lo manifestado por Machado (1996) que encontró que el déficit de humedad del suelo, influye negativamente en el crecimiento de los pastos, provocando reducción del área foliar, de la actividad fotosintética y, por consiguiente, de la producción de MS. Los resultados manifestados anteriormente también contrastan con lo dicho por Alencar (2007) y Oliveira (2007) quienes exponen que el aumento de láminas de riego incrementa la producción de materia seca de los pastos; al considerar que el volumen de agua aplicado en la investigación respondían a los cálculos de la ETc del pasto king grass morado en cada una de sus etapas de crecimiento, es decir el pasto no tenía un desbalance hídrico y mostro una alta producción de materia seca.

La eficiencia hídrica en función a la producción de pasto determinó la existencia de variación significativa entre los tratamientos $(\mathrm{p}<0.05)$ donde el tratamiento lámina de riego al $80 \%$ de la ETc prevalece con el mayor promedio, $61.16 \mathrm{~kg}$. de pasto por $\mathrm{m}^{3}$, esto quiere decir, que para producir un kilogramo de pasto se necesita aplicar 16.35 litros de agua. Mientras que la menor eficiencia hídrica lo consiguieron los tratamientos lámina de riego al 100\% de la ETc y lámina de riego al 120\% de la ETc con 49.23 y $46.80 \mathrm{~kg}$ de pasto por $\mathrm{m}^{3}$ respectivamente, lo que significa, que para producir un kilogramo de pasto se necesita aplicar 20.31 y 21.37 litros de agua (Cuadro 4). El consumo y eficiencia hídrica por tratamiento del pasto king grass morado durante los 45 días de la investigación, muestra que el tratamiento lámina de riego al 120\% de la ETc, presenta el mayor consumo de agua por hectárea con 1132.40 metros cúbicos $\left(\mathrm{m}^{3}\right)$ por hectárea, seguido del tratamiento lámina de riego al $100 \%$ de la ETc con $951.30 \mathrm{~m}^{3}$ ha, mientras que el menor consumo lo consiguió el tratamiento lámina de riego al $80 \%$ de la ETc con $749.40 \mathrm{~m}^{3}$ ha.

Esto quiere decir que la eficiencia hídrica de pasto es mayor cuando las láminas de agua que se aplican son menores a la necesidad hídrica de la planta, debido a una mejor distribución de asimilados en la planta en especial de la parte aérea, resultados que contrastan con lo manifestado por Ñústez et al. (2009) donde manifiestan que la asignación de asimilados en las plantas resulta de la interacción de condiciones climáticas, prácticas culturales y genotipo.

Cuadro 3. Promedio de porcentaje de materia seca del pasto king grass morado

\begin{tabular}{lc}
\hline Tratamientos & $\begin{array}{c}\text { Materia seca } \\
\text { (\%) }\end{array}$ \\
\hline Lámina de riego al $80 \%$ de la ETc & $18.19 \mathrm{a}$ \\
Lámina de riego al $100 \%$ de la ETc & $19.80 \mathrm{a}$ \\
Lámina de riego al $120 \%$ de la ETc & $19.86 \mathrm{a}$ \\
\hline CV (\%) & 5.60 \\
\hline
\end{tabular}

Medias seguidas con la misma letra, no difieren estadísticamente (Tukey, $\mathrm{p}>0.05$ ).

El pasto king grass morado regado con una lámina de riego al $120 \%$ de la ETc, constituye una modalidad que mejora el rendimiento productivo y rentable del cultivo, lo cual significa para el productor agropecuario mayor cantidad de alimento para el ganado utilizando la misma superficie de terreno pero con mayor uso del agua, resultados que coinciden con Medrano et al. (2008) quienes expresan que, mejorar la producción vegetal es compatible con la economía del agua, pero esto requiere más conocimientos y tecnología que se debe desarrollar para hacer más sostenible la producción de alimentos y el abastecimiento de las poblaciones. La producción agrícola mundial ha aumentado en paralelo a los

Cuadro 4. Consumo y eficiencia hídrica del pasto king grass morado

\begin{tabular}{lrcc}
\hline \multirow{2}{*}{ Etapas del cultivo } & \multicolumn{3}{c}{ Tratamientos } \\
\cline { 2 - 4 } & \multicolumn{1}{c}{ T1 } & T3 \\
\hline Etapa I & 153.60 & 198.90 & 238.80 \\
Etapa II & 171.50 & 222.10 & 257.20 \\
Etapa III & 424.30 & 530.30 & 636.40 \\
Volumen total $\left(\mathrm{m}^{3} \mathrm{ha}^{-1}\right)$ & 749.40 & 951.30 & 1132.40 \\
Rendimiento forraje verde $\left(\mathrm{kgha}^{-1}\right)$ & 45833.33 & 46833.33 & 53000.00 \\
Eficiencia hídrica $\left(\mathrm{kg} \mathrm{m}^{3}\right)$ & $61.16 \mathrm{a}$ & $49.23 \mathrm{~b}$ & $46.80 \mathrm{~b}$ \\
\hline CV $(\%)$ & \multicolumn{3}{c}{11.38} \\
\hline
\end{tabular}

Medias seguidas con la misma letra, no difieren estadísticamente (Tukey, $\mathrm{p}>0.05$ ). 
aumentos de población sin apenas incrementar la superficie cultivada. El aumento de producción global de alimentos viene totalmente ligado al incremento de la superficie regada, y esta tendencia «universal» se mantiene o se acentúa en los últimos años. El riego se hace cada vez más imprescindible para obtener una producción más regular y predecible de los cultivos.

\section{Conclusiones}

$\mathrm{L}$ a producción de forraje verde del pasto king grass morado está relacionado con los volúmenes de agua que se aplique. El contenido de materia seca está relacionado con las necesidades hídricas reales del pasto, factores ambientales y labores de manejo del cultivo. La eficiencia hídrica del pasto king grass morado es mayor cuando se aplica láminas de riego menores a la ETc. Las condiciones agroclimáticas de la zona del experimento favorecen la producción del pasto.

\section{Bibliografía}

Alencar, C. (2007). Produção de seis gramíneas forrageiras tropicais submetidas a diferentes lâminas de água e doses de nitrogênio, na região Leste de Minas Gerais. Tese (Doutorado em Engenharia Agrícola) - Universidade Federal de Viçosa, Viçosa, MG, Brasil. 121f.

Alencar, C., Cunha, F., Martins, C., Cóser, A., Rocha, W., Araújo, R. (2009). Irrigação de pastagem: atualidade e recomendações para uso e manejo. R. Bras. Zootec., 38: 98-108, (supl. especial).

Dávila, C. y Urbano, D. (2005). Uso de pastos de corte en los sistemas intensivos. En González, C. y Soto, E. (Eds). Manual de Ganadería Doble Propósito. Editorial Astro Data, Maracaibo, Venezuela. p. 193-198.

Dourato-Neto, D., Fancelli, A., Muller, M. (2002). Manejo da irrigação de pastagens. In: Simpósio dobre manejo de pastagens, 19. Piracicaba. Anais. Piracicaba: FEALQ. p. 189-216.

Doorembos, J. y Kassan, A. (1986). Efectos del agua sobre el rendimiento de los cultivos. Riego y Drenaje \#33. Roma, FAO. 178 p.

Ecuador-CEPAL. (2012). Diagnóstico de la Estadística del Agua en Ecuador. Informe final. Comisión Económica para América Latina y el Caribe. Ecuador. p.13.

Fraga, S., Santillán, B. (2008). Evaluación integral del complejo suelo-planta en una unidad lechera, con silvopastoreo, en la provincia La Habana. Cuba. Revista Cubana de Cienc. Agríc. 42(4): 403-408.

Gargantini, P., Hernández, F., Vanzela, L. (2005). Irrigação e adubação nitrogenada em capim mombaça na região Oeste do Estado de São Paulo. In: Congresso Nacional De Irrigação E Drenagem, 15. Teresina. Anais.

Herrera, J., González, F., Zamora, E. (2010). Coeficientes de cultivo $(\mathrm{Kc})$ del king grass para diferentes épocas del año y edad de la planta. Revista Ciencias Técnicas Agropecuarias 19(1): 44-49.

Idso, S. (1983). Stomatal regulation of evaporation from well-watered plant canopies: A new synthesis. Agrie. Meteorol.; 29(3): 213-217.

Lage, M., Bamouh, A., Karrou, M., El Mourid, M. (2003). Estimation of rice evapotranspiration using a microlysimeter technique and comparison with FAO Penman-Monteith and Pan evaporation methods under Moroccan conditions. Agronomie 23:625-631.

Machado, R. (1996). Dinámica de algunos indicadores morfológicos y estructurales de Andropogon gayanus CIAT-621, bajo condiciones de manejo intensivo. Efecto de la época y el año. Pastos y Forrajes 19:121-127.

Malek, E. (1987). Comparison of alternative methods for estimating ETp and evaluation of advection in the Bajgab area of Iran. Agricultural and Forest Meteorology 39:185-192.

Medrano, H., Bota, J., Cifre, J., Flexas, J., Ribas-Carbó, M. y Gulías, J. (2008). Eficiencia en el uso del agua por las plantas. Revista Investigaciones Geográficas (Esp), (43), 63-84.

Navejas, J., Nieto, A., Fraga, H., Rueda, E., Ávila, N. (2011). Comparación de métodos para estimar la evapotranspiración en una zona árida citrícola del Noroeste de México. Tropical and Subtropical Agroecosystems 13(2), 147-155.

Ñústez, C., Santos, M. y Segura, M. (2009). Acumulación y distribución de materia seca de cuatro variedades de papa (Solanum tuberosum L.) en Zipaquirá, Cundinamarca (Colombia). Rev. Fac. Nac. Agron. 62(1), 4823-4834.

Oliveira, J. (2007). Produção de duas gramíneas tropicais submetidas a diferentes lâminas de água e doses de nitrogênio e potássio no Estado do Tocantins. Tese (Doutorado em Engenharia Agrícola) - Universidade Federal de Viçosa, Viçosa, MG, Brasil. 121f.

Souza, E., Isepon, O., Alves, J. (2005). Efeitos da irrigação e adubação nitrogenada sobre a massa de forragem de cultivares de Panicum maximum Jacq. Revista Brasileira de Zootecnia 34(4), 1146-1155. 